\title{
Punctuated equilibrium by computer
}

Can a mathematical model of evolution dealing with species rather than their members as the substrate of natural selection accurately represent reality? Probably not, but it is suggestive and stimulating.

THAT physicists are itching to take over biology is now well attested. People are forever suggesting explanations for the fine details of cellular behaviour, from the ratchetlike behaviour of muscle fibres to the selfassembly of microtubules and even the tendency of lipid bilayer membranes to form cylinders with $\mu \mathrm{m}$ dimensions. But surely only a brave physicist would take on Darwin on his home ground, the theory of evolution, let alone Gould and Eldridge on punctuated equilibrium, at this early stage in the takeover game. That might be everybody's expectation. Predictably, it has now been falsified.

The guiding spirit behind the latest flurry of activity appears to be Per Bak, at the Brookhaven National Laboratory. Some years ago, with $\mathrm{C}$. Tang and $\mathrm{K}$. Wiesenfeld, he offered the world the sandpile as a model of what he called the self-organized critical behaviour of a dynamical system (Phys. Rev. Lett. 58, 381; 1987). The point of that model is that it represents a system far from equilibrium (which would be a layer of sand with a horizontal flat surface). If the sandpile is in the critical condition, the addition of a few grains of sand (in principle, just one of them) to the apex will trigger the formation of avalanches on the sides of the pile until a new position of equilibrium, perhaps metastable, is reached.

What has that to do with evolution, punctuated or otherwise? Well, may it not be reasonable to think of the long periods of evolutionary stasis as the times when sandpiles are slowly growing, and the avalanches triggered in a flurry at criticality as the bursts of speciation events that show up in the fossil record...? Whatever the truth of the matter, there has been a minor avalanche of articles on the theme since last December, all of which acknowledge their inspiration by Stuart Kauffman, the physician turned theoretical biologist at the Sante Fé Institute, whose book The Origins of Order was haughtily panned last year (Nature $\mathbf{3 6 5}$, 704; 1993).

The sandpile, of course, is not a good model for evolution. Bak and Kim Sneppen from the Niels Bohr Institute, explained that at the end of last year (Phys. Rev. Lett. 71, 4083-4086; 1993). Instead, they use points along the length of a line to represent the species in an entire ecosystem. This leads, the authors acknowledge, to the crudest of all possible models. A species is, after all, a collection of its individual members upon whom alone natural selection exerts its evolutionary influence. Representing an entire species by a single entity and ascribing to it a "fitness" which is a scalar number, which is what Bak and Sneppen did, will drive many evolutionists to despair.

Bak and Sneppen talked their way around that difficulty last year. The idea is that a species in a reasonably comfortable (or stable) condition may be likened to a complex physical system with many metastable states. The common physical analogy is that of a spin-glass, essentially a randomly distributed system of magnetic spins interacting with at least their neighbours and allowed to find their lowest energy.

The possible states of such a system include at least an infinite number in which magnetized patches of arbitrary orientation are embedded in each other. Each of these is a candidate for stability relative to the states obtained by reversing the direction of an arbitrary spin in the spin-glass as a whole, but that is no assurance that the energyminimum is the lowest of all possible. For what it is worth, the same analogy is used (at least in words) for modelling the conditions of neural networks.

That is the underlying model for the fitness of a species, taken to be the negative of its energy. Ideally, species would remain at a local maximum of the "fitness landscape", but the occurrence of mutations with the succession of the generations might easily drive them away. Extinction might be one consequence, but otherwise their best refuge would be a neighbouring peak in the landscape with, preferably, greater fitness.

So how to carry out the arithmetic? There are $N$ species, each represented by a point along a line (whose chief purpose is that each point should have two neighbours). Each species (point) is randomly assigned a fitness-number, chosen (without loss of generality, as mathematicians say) to be between 0 and 1. Following the analogy of the spin-glass, Bak and Sneppen go on to argue that the evolutionary barriers (the impediments to further change) will be least for those species whose fitness is represented by the smallest number, so that the fitness number is taken as a measure of the barrier to further change.

The next step is to choose the species with the smallest fitness and to reassign it another random number. To allow for the circumstance that a change in the competence of one species will often change the prospects for others, Bak and Sneppen also reassign random numbers to the two neighbouring species in the line. To allow for what may be happening independently to other species, they also select a certain number of other points along the line and randomly reassign them random numbers.

The most interesting conclusion from the numerical results is that the initial conditions are irrelevant to the final outcome. The system settles down to one in which the barrier height (or fitness) is high for most species. If, in the reassignment of fitness, a small number appears, the species concerned will be quickly dragged back to a conditions of high fitness through a series of readjustment of the species and its neighbours. So the analogy with the sandpile applies. The wave of readjustments in one species and its neighbours is like an avalanche down the slope of the pile, whose steady state is therefore a state of criticality.

That was Bak and Sneppen last December. The same authors and Henrick Flyvbjerg of Princeton, in the same issue of the same journal $(71,4087-4090 ; 1993)$, described a variant of the model in which the two nearest neighbours of the least fit species were not modified at each time step, but a fixed number were. (That is equivalent to the assumption that a change in the fitness of one species affects a fixed number of others chosen randomly - the line is unnecessary.) That, with a little approximation, can be dealt with analytically. The results confirm what the simulations show. And that argument has now been carried further by Jan de Boer from the State University of New York at Stonybrook and a group of associates (Phys. Rev. Lett. 73, 906-909; 1994) to show that the evolutionary avalanches are just what Gould and Eldridge need to punctuate evolutionary equilibrium.

How convincing is all this? There is no doubt that the authors have found a model that reproduces punctuated equilibrium, but whether it represents the real world is less obvious. One drawback is that it allows neither for the extinction of species nor for their divergence into two or more. Another is that there is no telling whether the real evolutionary landscape is like that supposed in the model. (There are one or two references to "coordinated mutation" to account for the evolution of structures such as wings that would raise some biologists' hackles.) But the authors are right to say that previous treatments of self-organized criticality have shown these systems to be robust against detailed changes of the model. If a simple problem can be handled, a more complicated one may well give the same result. So the exercise should not be forgotten.

John Maddox 